\title{
Proposta de Algoritmo para Gerenciamento Pelo Lado Demanda em ResidênCias Através do CORTe De SMART Plugs
}

\author{
Eliane Silva CUSTÓdio ${ }^{1}$, LUCAS RobERTO FERREIRA ${ }^{1}$, LUCIANO CAVALCANTE SIEBERT ${ }^{1}$, EdUARDO KAZUMI

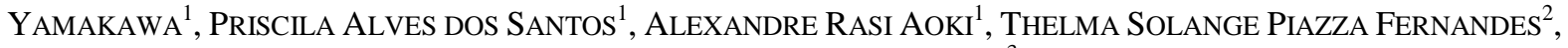 \\ ESDRAS ELIWAN MARTINS LEITE ${ }^{3}$
1. LACTEC - Instituto de Tecnologia para o Desenvolvimento, Departamento de Eletricidade, Divisão de Sistemas Elétricos
BR 116 - Km 98 - No 8813, Centro Politécnico da UFPR, Curitiba - PR.
E-mails: eliane.custodiodlactec.org.br, lucas.ferreirallactec.org.br, luciano.siebertelactec.org.br, eduardodlactec.org.br, priscila.santoselactec.org.br, aokidlactec.org.br

2. UFPR, Universidade Federal do Paraná

Francisco H. dos Santos - Centro Politécnico, Curitiba - PR

E-mail:thelmadeletrica.ufpr.br

3. LIGHT S.E.S.A, Gerência de Tecnologia e Inovação

Av Marechal Floriano 168, Rio de Janeiro - RJ

E-mail: esdras.leitelight.com.br

\begin{abstract}
Due to the increase of electricity demand every year and rising problems in its distribution, it is a need for the utilities to find ways to supply this demand with techniques beyond the usual infrastructural growth. One possibility is to use demand side management, including actions concerning load shifting, e.g. actions to change the time pattern consumption of equipment from peak to off-peak hours encouraged by Time-Of-Use tariffs. To support the customers in these time pattern consumption changes, aiming benefits to both utility and customers, it is being lately often discussed the use of smart plug as a measuring and shedding tool, supported by algorithms that provide the need automation and optimization. The algorithm presented in this paper was able to provide satisfactory results, indicating when the customer should avoid using each smart plug, as well as avoiding energy waste, through the shed of energy consumption in standby.
\end{abstract}

Keywords—Demand Side Management, Load Shifting, Smart Plugs, White Tariff, Power Systems.

Resumo- Com o aumento da demanda de energia a cada ano e consequentes dificuldades no processo de distribuição da mesma, é necessidade das concessionárias encontrar saídas para conseguir suprir esse aumento, procurando técnicas além do aumento da infraestrutura. Uma dessas saídas é o gerenciamento pelo lado da demanda, que engloba diversas técnicas incluindo o deslocamento de carga, ou seja, ações para realizar o deslocamento da utilização de equipamentos do horário de pico para outro horário, incentivadas através de tarifas variáveis no tempo. Para dar suporte ao cliente nessas ações, visando benefícios para a concessionária e também reduções de gastos de energia do cliente, ultimamente vem sido bastante discutida a utilização de smart plugs como ferramenta de medição e de corte, auxiliada por algoritmos que possibilitem a automação e otimização das mesmas. $\mathrm{O}$ algoritmo desenvolvido no presente trabalho apresentou resultados satisfatórios, indicando os momentos que o consumidor deve evitar o consumo de energia em cada smart plug assim como auxiliar na redução do desperdício de energia, atuando no corte do consumo de aparelhos em standby.

Palavras-chave— Gerenciamento pelo Lado da Demanda, Deslocamento de Carga, Tomadas Inteligentes, Tarifa Branca, Sistemas de Potência.

\section{Introdução}

Conforme Falcão (2010), smart grid deve ser entendido mais como um conceito do que uma tecnologia ou equipamento específico. O smart grid carrega a ideia da utilização intensiva de tecnologia de informação e comunicação na rede elétrica, através da possibilidade de comunicação do estado dos diversos componentes da rede, o que permitirá a implantação de estratégias de controle e otimização da rede de forma muito mais eficiente que as atualmente em uso.

O monitoramento em tempo real do desempenho da rede pode melhorar a confiabilidade do sistema, reduzir apagões e aumentar o retorno financeiro sobre os investimentos na rede. Essas mudanças na demanda e oferta exigem sistemas inteligentes para gerenciar de forma eficiente essa rede cada vez mais complexa (Hashmi, 2011).

Segundo Gellings (1985), gerenciamento pelo lado da demanda (DSM - Demand-Side Management) é o planejamento e implementação de atividades para influenciar o uso de eletricidade do consumidor de maneira que produza mudanças desejadas na curva de carga de um sistema elétrico. O DSM é uma ferramenta útil para melhorar a eficiência energética e reduzir o consumo total de eletricidade.

Uma ferramenta para facilitar a efetividade de ações de DSM, principalmente no âmbito residencial, é o smart plug. Consiste em um medidor de energia com baixo custo de fabricação, tamanho reduzido e com um canal de comunicação bidirecional direta 
com o medidor central de uma residência cuja função é medir o consumo individualizado de cada carga. Esse dispositivo também possibilita o corte via comando remoto, agendamento ou sobrecarga.

Fernandes et al. (2011) apresentou uma abordagem para gerenciar diretamente o consumo de cargas em uma residência utilizando algoritmos genéticos e fazendo uso de um sistema SCADA desenvolvido pelos autores. Essa gestão do consumo foi feita reduzindo ou limitando cargas para manter o consumo de energia abaixo de um limite determinado de acordo com a estratégia do consumidor, e também levando em conta a geração local baseada em fontes renováveis de energia, preços, solicitações de fornecedores e consumidores.

Barbato et al. (2011) apresenta um sistema para prever o uso de eletrodomésticos, a fim de fornecer automaticamente as entradas de mecanismo de gerenciamento de energia elétrica, da mesma maneira que um usuário poderia fazer. A arquitetura é composta por sensores de medição acoplados aos eletrodomésticos e por uma central de armazenamento. Os dados fornecidos pelos sensores foram processados a cada 24 horas para prever quais os dispositivos serão utilizadas no dia seguinte, em que momento do dia e durante quanto tempo. Esta informação representou apenas os parâmetros de entrada exigidos pelos sistemas de gestão da carga de demanda, evitando assim complexas configurações manuais pelo usuário.

Em Di Giorgio et al. (2011) é proposta uma estratégia de controle que proporcionou uma gestão eficiente da energia elétrica em um ambiente doméstico. O problema foi contextualizado como um do tipo de programação linear binária, onde a saída é o melhor momento para ligar eletrodomésticos inteligentes, sob uma restrição de potência máxima de consumo. Essa potência máxima leva em conta a potência contratada e a previsão de consumo de cargas não planejáveis. Esta formulação permitiu analisar cenários relevantes do consumidor varejista: o primeiro cenário leva em consideração a otimização de economia de energia em caso de diferentes tarifas contratadas e o segundo cenário leva em consideração um plano de gestão pelo lado da demanda. As simulações foram realizadas em casos relevantes, com base em perfis de carga reais fornecidos pela empresa de eletrodomésticos inteligentes Electrolux e em tarifas de energia sugeridos pela concessionária norte-americana Edison. Os resultados forneceram uma prova do conceito relacionado aos benefícios do uso de um sistema de controle de energia e de um plano de DSM, tanto para economia do consumidor quanto para o funcionamento eficaz do sistema de distribuição de energia.

Conejo et al. (2010) também descreveu um modelo de otimização para ajustar o nível de carga horária de um determinado consumidor em resposta a preço da eletricidade naquela hora. $\mathrm{O}$ objetivo do modelo foi minimizar a conta do consumidor. O problema se resumiu em algoritmo de programação linear simples que pode ser implementado em um sistema maior de controle pelo lado da demanda. A comunicação bidirecional entre o consumidor e o fornecedor foi um fator importante para desenvolvimento desse trabalho.

$\mathrm{O}$ algoritmo proposto neste artigo tem como objetivo o gerenciamento de tomadas inteligentes (smart plugs) para otimizar a curva de carga e o gasto com energia elétrica do consumidor de acordo com uma tarifa variável no tempo. As técnicas de DSM utilizadas são o deslocamento de carga através do corte de smart plugs em horário de pico e a conservação estratégica através da diminuição do consumo de aparelhos em modo standby.

As seções apresentadas a seguir estão dividas na seguinte ordem: uma breve explicação sobre a tarifa branca, considerada nas simulações (2); a metodologia de gerenciamento pelo lado da demanda desenvolvida (3); o estudo de casa utilizando dados reais medidos pelos smart plugs (4); resultados e análises (5) e por fim a conclusão do trabalho (6).

\section{Tarifa Branca}

Em novembro de 2011 foi aprovada pela Agência Nacional de Energia Elétrica (ANEEL) a implantação da tarifa branca na estrutura tarifária aplicada ao setor de distribuição de energia brasileiro, incluindo clientes residenciais e comerciais, conforme discussões realizadas na audiência pública $n^{\circ} 120 / 2010$ e presentes no módulo 7.1 do Procedimento de Regulação Tarifária da ANEEL (ANEEL, 2013).

Tal tarifa é do tipo Time-Of-Use (com postos tarifários fixos refletindo situações típicas de relação entre fornecimento e demanda), monômia, de adesão voluntária, com três preços de acordo com os postos tarifários:

- Ponta - período de 3 horas consecutivas diárias, exceção feita aos sábados, domingos e feriado;

- Intermediária - período formado pela hora imediatamente anterior e pela hora imediatamente posterior ao período de ponta, totalizando 2 horas;

- Fora de ponta - período composto pelas horas complementares aos períodos de ponta e intermediária.

O sistema de DSM proposto nesse trabalho tem como objetivo de propor aos clientes um agendamento do consumo de energia de equipamentos conectados a smart plugs para que a utilização da tarifa branca seja um elemento para impulsionar a eficiência do consumo de energia e da rede, assim como possibilitar ganhos financeiros pelos clientes.

\section{Metodologia}

A presente seção trata da metodologia do algoritmo usado para fazer o deslocamento de carga e o corte de consumo de aparelhos em standby, tendo como entrada o perfil de consumo do consumidor (medido através dos smart-plugs implantados numa residên- 
cia); a tarifa usada e o nível de redução desejado para o deslocamento. Os parâmetros de entrada, portanto são:

perfil $_{\text {consumo }}$ - perfil de consumo médio, gerado através da curva de carga de 4 dias, neste caso quatro segundas-feiras, em Watts;

$\boldsymbol{t}_{\boldsymbol{b}}$ - valor do posto tarifário de nível baixo, em reais;

$\boldsymbol{t}_{\boldsymbol{i}}$ - valor posto tarifário de nível intermediário, em reais;

$\boldsymbol{t}_{\boldsymbol{a}}$ - valor posto tarifário de nível alto, em reais;

$\boldsymbol{h} \boldsymbol{b}_{\boldsymbol{i}}, \boldsymbol{h} \boldsymbol{b}_{\boldsymbol{f}}$ - horário de início e de término, respectivamente, do posto tarifário de nível baixo, em minutos;

$\boldsymbol{h} \boldsymbol{i}_{\boldsymbol{i}}, \boldsymbol{h} \boldsymbol{i}_{\boldsymbol{f}}$ - horário de início e de término, respectivamente, do posto tarifário de nível intermediário, em minutos;

$\boldsymbol{h} \boldsymbol{a}_{\boldsymbol{i}}, \boldsymbol{h} \boldsymbol{a}_{\boldsymbol{f}}$ - horário de início e de término, respectivamente, do posto tarifário de nível alto, em minutos. $\Delta \boldsymbol{t}$ - período de amostragem dos dados dos smart plugs, em minutos;

$\boldsymbol{n}=\frac{(\mathbf{2 4 * 6 0 )}}{\Delta t}-$ quantidade de períodos de aquisição durante um dia;

parametro $_{\text {media }}$ - parâmetro para deslocar a linha de corte do standby;

parametro $_{\text {redução }}$ - parâmetro de nível de redução de energia.

A primeira análise feita é o gasto em standby. A

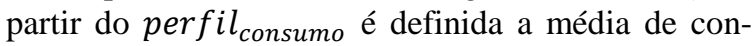
sumo para cada dia, e assim determinada à linha de corte do standby, definida por:

linha $_{\text {corte }}=$ media $*$ parametro $_{\text {media }}$

Sujeito a:

$0 \leq$ linha $a_{\text {corte }} \leq 50 \mathrm{~W}$

A inferência dos horários previstos de consumo de aparelhos em modo standby é definido por:

locais $_{\text {standby }}=$ pefil consumo $_{\text {colinha }}$ corte $_{\text {con }}$

Na sequencia é calculado o valor máximo a ser economizado pelo algoritmo (deslocamento de carga e conservação estratégica de carga), deslocando todo o consumo no momento de pico e pico intermediário para horários de tarifa baixa, através de:

$$
\begin{aligned}
\text { consumo }_{\text {pico }}= & \sum_{i=h i_{i}}^{h i_{f}} \operatorname{perfil}_{\text {consumo }}(i) * t_{i} \\
& +\sum_{j=h a_{i}}^{h a_{f}} \operatorname{perfil}_{\text {consumo }}(j) * t_{a}
\end{aligned}
$$

$$
\begin{aligned}
\text { valor }_{\text {economizado }}= & \text { consumo }_{\text {pico }} \\
& -\sum_{\substack{h b_{f} \\
\text { phb }}} \text { perfil } \\
& + \text { locaissumo }_{\text {standby }} * t_{b}
\end{aligned}
$$

Desse total é selecionada a porcentagem desejada para redução, o que resultará na meta de redução que será considerada na próxima fase do algoritmo.

$$
\begin{aligned}
\text { meta }= & \text { valor }_{\text {economizado }} \\
& * \text { parametro } o_{\text {redução }}
\end{aligned}
$$

Na fase de deslocamento de carga do algoritmo, primeiramente é realizada a etapa de "liberação" dos smart plugs (inicialmente é considerado que todos serão bloqueados na tarifa intermediária e alta) que impactem em um menor custo na tarifa de pico. Essa "liberação" é para todo o período do corte e é realizada até o limiar do alcance da meta desejada, conforme a Figura 1. Se a liberação dos smart plugs (por exemplo, $\mathrm{SP} 2_{1}$ e $\mathrm{SP} 1_{1}$ ) atinge exatamente o valor da meta, é finalizada a análise, caso contrário é necessário à realização da etapa de redução dos limites.

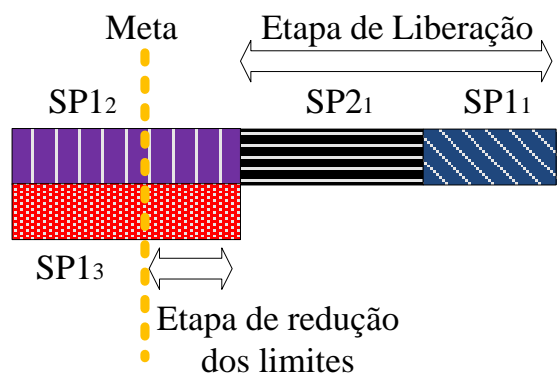

Figura 1. Elaboração do corte nos smart plugs.

Essa é realizada de forma simultânea em todos os demais smart plugs que não foram liberados e realizará, iniciando através da tarifa média $(\mathrm{Tm})$ ou intermediária, a diminuição do período de corte até que o custo seja igualado à meta, conforme Figura 2. Caso a liberação de todos smart plugs no período Tm não seja suficiente para atingir a meta, é realizada a diminuição do período na tarifa alta (Ta).

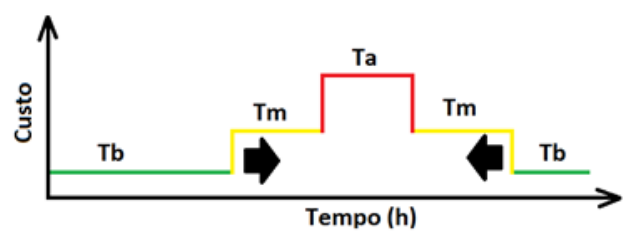

Figura 2. Redução dos limites de corte tomando como exemplo a tarifa branca com três postos tarifários. 


\section{Estudo de Caso}

No estudo de caso, descrito nesta seção, utilizou-se dados reais de consumo de energia de uma residência, obtidos através da instalação de quatro smart plugs. Os dispositivos conectados são: $\mathrm{SP} 1_{1}-\mathrm{um}$ computador e dois monitores; $\mathrm{SP}_{2}$ - um computador, um monitor, duas impressoras e um scanner; $\mathrm{SP}_{3}$ - uma TV, um XBOX 360, um receptor de TV a cabo, um aparelho blue-ray e um receptor Akai; $\mathrm{SP} 2{ }_{1}$ - lavadora de roupas.

As medições feitas pelos smart plugs foram realizadas durante o período de uma semana, porém para efeitos das simulações que serão apresentadas, considerou-se que os quatro primeiros dias da semana (segunda - terça - quarta - quinta), representassem todos segunda-feira de semanas subsequentes. Visou-se assim apresentar um perfil médio de consumo de um mês para o primeiro dia útil da semana.

Através da manipulação da curva de carga supracitada, foram elaborados diversos cenários para comprovação de funcionalidade do algoritmo proposto.

\subsection{Cenário 1}

O primeiro cenário é o perfil de consumo normal da residência, conforme a Figura 3 . Nota-se que SP2 1 tem o funcionamento fora do horário de pico, sendo usado somente no turno da manhã, o $\mathrm{SP} 1_{1}$ tem um consumo baixo durante a parte da madruga e nos outros horários há um consumo sem grandes variações repentinas. Os demais smart plugs têm um funcionamento mais acentuado durante o horário de pico.

\subsection{Cenário 2}

O objetivo do cenário 2 é deslocar o funcionamento da lavadora de roupa para o horário de pico, compreendido pelos períodos de postos tarifários intermediário e alto, simulando uma situação onde o morador da residência tenha chegado do trabalho e iniciado a lavagem das roupas. Isso transfere o consumo de energia do equipamento instalado no SP $2_{1}$, que antes era pela manhã, para o horário de pico, como apresenta a Figura 4.

\subsection{Cenário 3}

Este cenário tem como proposta distribuir mais igualitariamente o consumo dos smart plugs ao longo do dia. Mantém-se o uso da lavadora fora do horário de pico e é deslocado o $\mathrm{SP}_{2}$ do horário de pico. Com isso, somente o consumo de $\mathrm{SP} 1_{3}$ e $\mathrm{SP} 1_{1}$ foi considerado para a ponta.

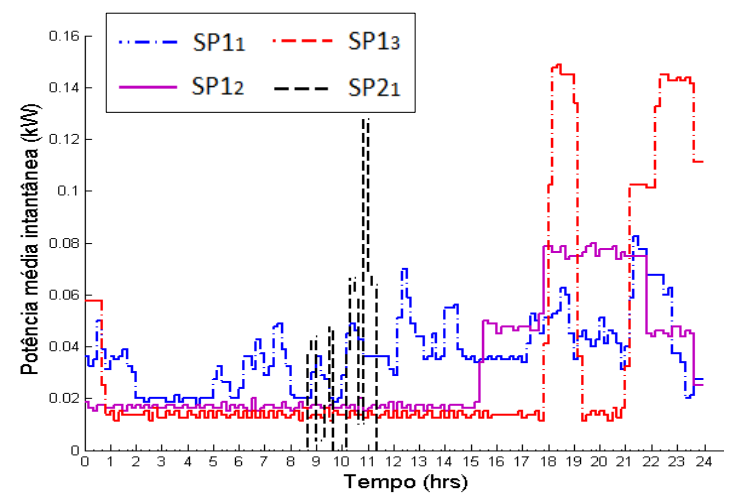

Figura 3. Perfil de consumo para o cenário 1.

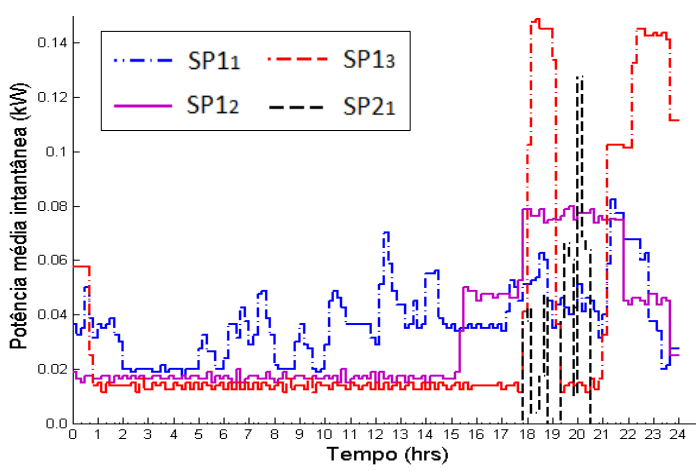

Figura 4. Perfil de consumo para o cenário 2.

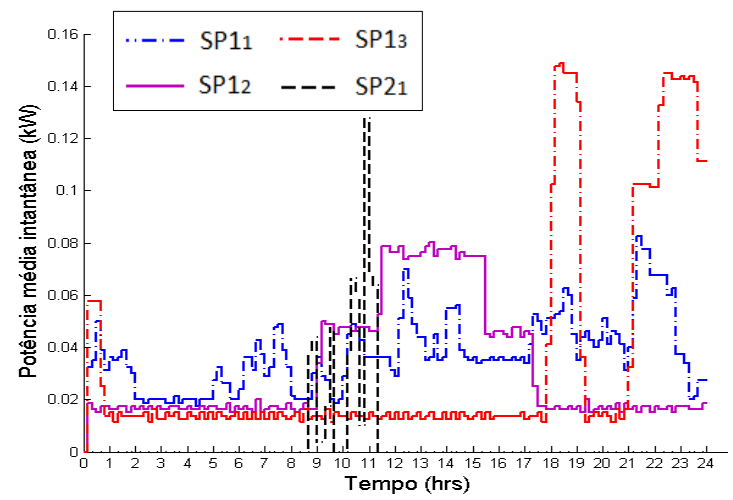

Figura 5. Perfil de consumo para o cenário 3.

\section{Resultados}

Neste capítulo são apresentados os resultados dos agendamentos para o cenário descritos no capítulo quatro. Nas simulações realizadas foi considerado um valor de 0,75 para o parametro $o_{\text {media }}$ e dois valores diferentes para o parametro $o_{\text {redução, um de }}$ redução de gasto de energia moderada $(50 \%)$ e outro de alta (80\%). A legenda das Figuras 6, 7 e 8 é apresentada abaixo:

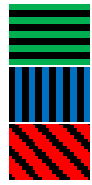

SP liberado

SP emstandby

SP cortado 
Redução Moderada
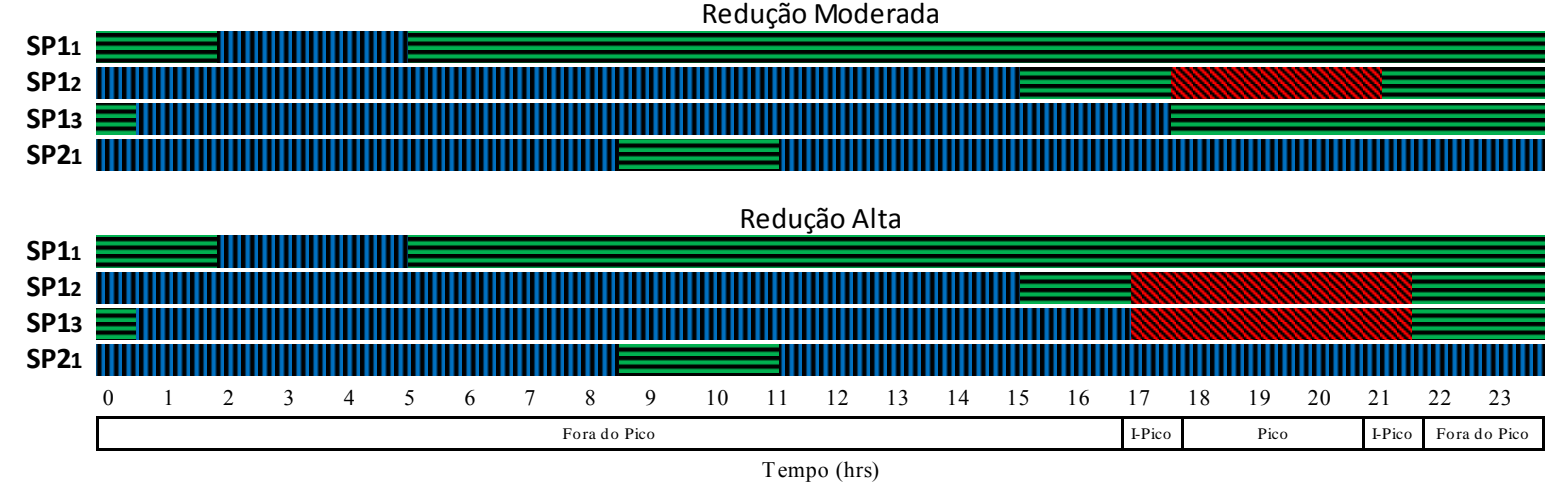

Figura 6. Agendamento dos smart plugs para o cenário 1.

Redução Moderada

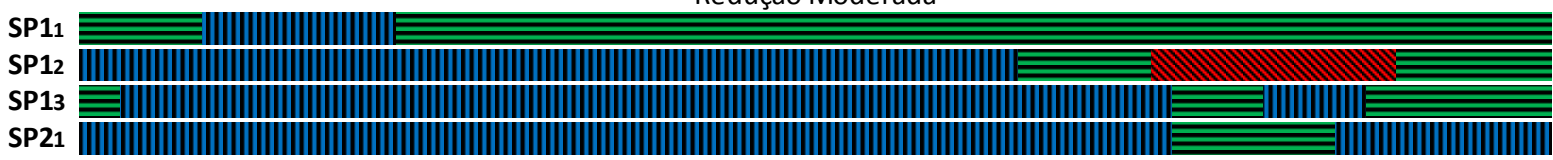

Redução Alta

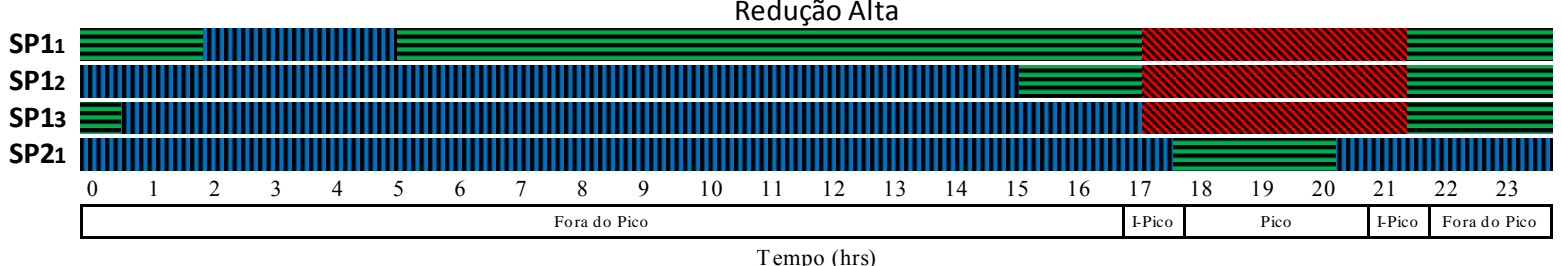

Tempo (hrs)

Figura 7. Agendamento dos smart plugs para o cenário 2.

Redução Moderada

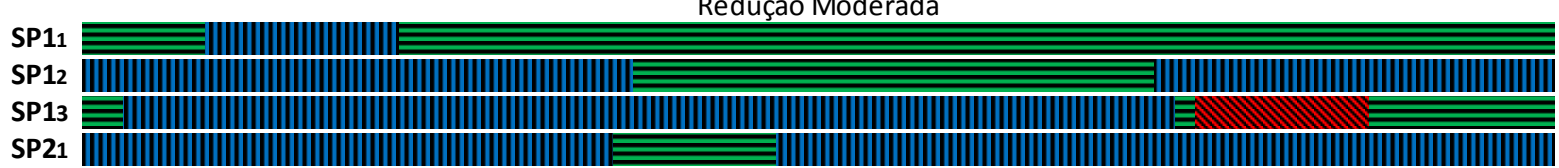

Redução Alta

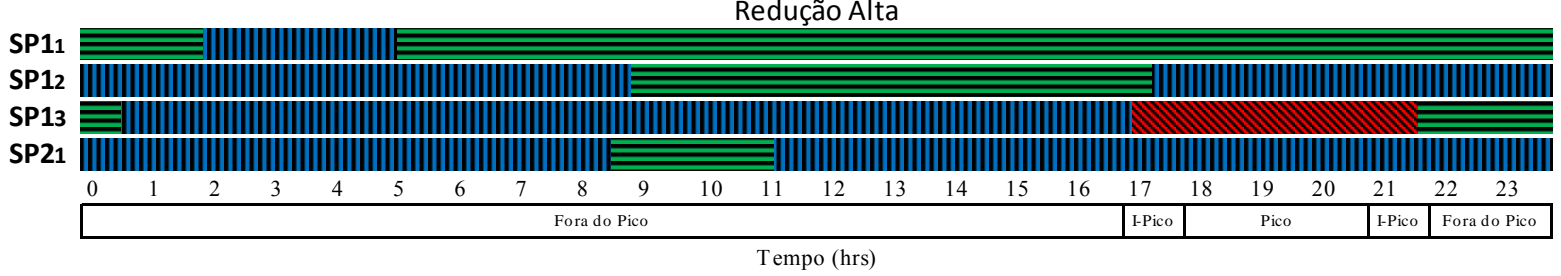

Figura 8. Agendamento dos smart plugs para o cenário 3.

\subsection{Cenário 1}

Conforme a metodologia implementada, na qual é realizada inicialmente a etapa de "liberação" dos smart plugs, nota-se que para uma redução moderada, conforme Figura 6, SP1 $1_{1}, \mathrm{SP} 1_{3}$ e $\mathrm{SP} 2_{1}$ foram liberados por completo. $\mathrm{SP} 1_{2}$, por sua vez, foi cortado por um período reduzido, atingindo assim a meta desejada. Para a redução alta, ocorreu a "liberação" por completo do SP1 $1_{1}$ e do SP2 $2_{1}$. Nesse patamar de redução, o corte de $\mathrm{SP} 1_{3}$ e $\mathrm{SP} 1_{2}$ representa a redução de energia desejada para se alcançar a meta.

Como apresentado na seção quatro, a inferência de consumo em standby não é influenciada pelo patamar de redução desejado, logo o corte para evitar standby é o mesmo em ambos os casos, exceto para o $\mathrm{SP}_{1}$. Isso acontece devido à prioridade que o corte por tarifa (deslocamento de carga) tem sobre o corte devido ao standby.

\subsection{Cenário 2}

A Figura 7 mostra que para o cenário 2, no patamar moderado de redução, o SP1 $1_{2}$ é cortado por um período mais longo do que quando comparado ao cenário 1. Isso ocorreu, pois, mesmo considerando o acréscimo de carga no horário de pico e consequente aumento na meta, o algoritmo foi capaz de atingi-la realizando a "liberação" de SP1 $1_{1}, \mathrm{SP} 1_{3}$ e SP2 $2_{1}$.

No caso da redução alta, a meta foi atingida através da "liberação" do SP2 $2_{1}$, que embora esteja no horário de pico não é realizado o corte, pois a devido ao empilhamento das curvas de cargas, o consumo de energia da máquina de lavar é menos significativo que as demais curvas. E o outro fator para atingir a meta foi o deslocamento de carga nos demais smart plugs, como apresenta a Figura 7. Analogamente ao cenário anterior, nos momentos fora de pico o corte 
para evitar gasto com standby se manteve igual nos dois patamares.

\section{$5.3 \quad$ Cenário 3}

Considerando um menor número de cargas no horário de pico, é visivelmente perceptível, conforme Figura 8, quando comparado as cenários 1 e 2, que os limites de corte diminuíram, e para o patamar de redução alta, a quantidade de smart plugs cortados no horário de pico foi menor. Como neste caso há somente os $\mathrm{SP} 1_{1}$ e SP1 $1_{3}$ com um consumo significativo no horário de pico, "liberou-se" o SP1 1 que apresentava menor consumo, em conjunto com os demais sem consumo, e limitou o deslocamento de carga do $\mathrm{SP}_{3}$ para satisfazer a meta de redução. O corte por standby se manteve igual, sendo somente sobreposto pelo deslocamento de carga.

\subsection{Análise Comparativa}

Comparando os três cenários conforme os níveis de redução, através da quantidade de minutos que se evitou com o gasto por standby e com o corte da energia no pico pela tarifa.

A Tabela 1 apresenta a duração, em minutos, do corte por standby e do deslocamento de carga, considerando a soma de todos os smart plugs em cada cenário. Portanto, para o caso do standby, como os cenários para a simulação utilizava as mesmas curvas de cargas, os resultados manteve-se com valores próximos em todos os cenários, a diferença ocorre devido ao fato de que o deslocamento de carga sobrepõe o standby no resultado final. Observa-se claramente também que quanto maior a carga no horário de pico, maior será a meta de corte e consequentemente maior será o deslocamento de carga, pois a meta está diretamente ligada com o perfil de consumo.

Tabela 1. Comparação dos cenários conforme os tempos de corte

\begin{tabular}{|c|c|c|c|}
\hline \multicolumn{2}{|c|}{} & $\begin{array}{c}\text { Total de corte por } \\
\text { standby [min] }\end{array}$ & $\begin{array}{c}\text { Deslocamento } \\
\text { de carga [min] }\end{array}$ \\
\hline \multirow{3}{*}{$\begin{array}{c}\text { Redução } \\
\text { Moderada }\end{array}$} & Cenário 1 & 3520 & 210 \\
\cline { 2 - 4 } & Cenário 2 & 3520 & 240 \\
\cline { 2 - 4 } & Cenário 3 & 3430 & 170 \\
\hline \multirow{3}{*}{$\begin{array}{c}\text { Redução } \\
\text { Alta }\end{array}$} & Cenário 1 & 3380 & 560 \\
\cline { 2 - 4 } & Cenário 2 & 3390 & 780 \\
\cline { 2 - 4 } & Cenário 3 & 3390 & 280 \\
\hline
\end{tabular}

\section{Conclusão}

Nesse artigo um algoritmo para gerenciamento pelo lado da demanda (deslocamento e conservação estratégica de carga) foi descrito e testado. A partir das simulações, realizadas com dados medidos por smart plugs em uma residência, mostraram que o algoritmo é capaz de fornecer propostas de agendamento do corte de smart plugs considerando tanto o perfil de consumo do cliente quanto suas preferên- cias. O aumento da eficiência do uso de energia na residência é endereçado através da inferência e posterior corte do consumo dos dispositivos em modo standby.

O algoritmo permite também: i) avaliação da aplicabilidade da tarifa branca no contexto do estudo de caso e ii) verificar a importância de sistemas automáticos para auxiliar o cliente na otimização do seu perfil de carga, através de automação residencial e algoritmos inteligentes.

Para trabalhos futuros recomenda-se, quanto ao algoritmo desenvolvido, uma análise mais criteriosa na determinação da linha de corte do standby e considerar possíveis agendamentos manuais nos smart plugs feitos pelo consumidor. Quanto à aplicação de algoritmos para gerenciamento pelo lado da demanda em residências recomende-se o estudo comparativo entre abordagens decentralizadas (nos smart plugs) e centralizadas e também um sistema de otimização multi-residência.

\section{Agradecimentos}

O presente trabalho foi apoiado financeiramente e institucionalmente pelo programa Smart Grid da concessionária Light.

\section{Referências Bibliográficas}

ANEEL (2013). Procedimentos de Regulação Tarifária - Submódulo 7.1.

Barbato, A.; Capone, A.; Rodolfi, M.; Tagliaferri, D.(2011). Forecasting the usage of household appliances through power meter sensors for demand management in the smart grid. IEEE International Conference on Smart Grid Communications (SmartGridComm), p. 404-409. DOI: 10.1109/SmartGridComm.2011.6102356

Conejo, A. J.; Moralae, J. M.; Baringo, L. (2010). Real-Time Demand Response Model. IEEE Transactions on Smart Grid, v. 1, n. 3, p. 236242. DOI: 10.1109/TSG.2010.2078843

Di Giorgio, A.; Pimpinella, L.; Quaresma, A.; Curti, S (2011). An event driven Smart Home Controller enabling cost effective use of electric energy and automated Demand Side Management. Mediterranean Conference on Control \& Automation (MED), p. 358-364.

Falcão, D. M. (2010). Integração de Tecnologias para Vialização da Smart Grid. III Simpóstio Brasileiro de Sistemas Elétricos.

Fernandes, F.; Sousa, T.; Silva, M. et al (2011). Genetic Algorithm Methodology applied to Intelligent House Control. IEEE Computational Intelligence Applications in Smart Grid.

Gellins,C. W. (1985). The Concept of Demand-Side Management for Electric Utilities. Proceedings of the IEEE, v. 73, n. 10, pp. 1468-1470. DOI: 10.1109/PROC.1985.13318

Hashimi, M (2011). Survey of smart grids concepts worldwide. 